\title{
Epitaxial interfaces between crystallographically mismatched materials
}

\author{
Steven C. Erwin, ${ }^{1}$ Cunxu Gao, ${ }^{2}$ Claudia Roder, ${ }^{2}$ Jonas Lähnemann, ${ }^{2}$ and Oliver Brandt ${ }^{2}$ \\ ${ }^{1}$ Center for Computational Materials Science, Naval Research Laboratory, Washington, DC 20375 \\ ${ }^{2}$ Paul-Drude-Institut für Festkörperelektronik, Hausvogteiplatz 5-7, 10117 Berlin, Germany
}

(Dated: December 18, 2018)

\begin{abstract}
We report an unexpected mechanism by which an epitaxial interface can form between materials having strongly mismatched lattice constants. A simple model is proposed in which one material tilts out of the interface plane to create a coincidence-site lattice that balances two competing geometrical criteria-low residual strain and short coincidence-lattice period. We apply this model, along with complementary first-principles totalenergy calculations, to the interface formed by molecular-beam epitaxy of cubic $\mathrm{Fe}$ on hexagonal $\mathrm{GaN}$ and find excellent agreement between theory and experiment.
\end{abstract}

PACS numbers: 81.15.Aa, 81.15.Hi, 68.55.-a, 68.37.Lp

A fundamental goal of materials science is to elucidate and exploit the physical principles that govern epitaxial growth [1, 2]. Some of these principles are wellestablished. For example, if the lattice constants of the film and substrate are close but not identical then a coherently strained film may grow up to a critical thickness, beyond which misfit dislocations relieve the strain [3]. Alternatively, a film and substrate having lattice spacings close to an integer ratio $m / n$ may form an epitaxial interface described by a coincidence lattice [4, 5].

In this Letter we report a new and unexpected mechanism by which epitaxial films can grow on substrates having, in principle, an arbitrary lattice mismatch. We illustrate this mechanism experimentally by growing singlecrystal Fe on $M$-plane GaN. The Fe grows in an unusual crystallographic orientation with a very high Miller index, $\mathrm{Fe}(205)$. We develop a simple theoretical model which, when complemented with total-energy calculations, correctly predicts this exact orientation as well as the singledomain nature of the film. Finally, we use our model to propose a new strategy for growing nonpolar epitaxial $\mathrm{GaN}$ films on high-index Si substrates.

The epitaxial growth of both $\mathrm{GaN}$ and Fe was performed in a custom-built molecular-beam epitaxy system equipped with solid-source effusion cells for $\mathrm{Ga}$ and $\mathrm{Fe}$. Active nitrogen was provided by a radio-frequency $\mathrm{N}_{2}$ plasma source. Nucleation and growth were monitored in situ by reflection high-energy electron diffraction. A 130-nm thick layer of $M$-plane $\mathrm{GaN}$ was first grown on a $4 \mathrm{H}$ $\mathrm{SiC}(1 \overline{1} 00)$ substrate under $\mathrm{Ga}$-stable conditions and a temperature of $720^{\circ} \mathrm{C}$. After growth of the GaN layer, excess $\mathrm{Ga}$ was desorbed prior to cooling down to $350^{\circ} \mathrm{C}$ for the deposition of Fe [6]. The Fe film grew at this temperature at a rate of $0.13 \mathrm{~nm} / \mathrm{min}$ to a final thickness of $27 \mathrm{~nm}$. The resulting $\mathrm{Fe} / \mathrm{GaN} / \mathrm{SiC}$ heterostructure was investigated by cross-sectional transmission electron microscopy (TEM) and convergent-beam electron diffraction (CBED) using a JEOL JEM-3010 operating at $300 \mathrm{keV}$. Electron backscat-

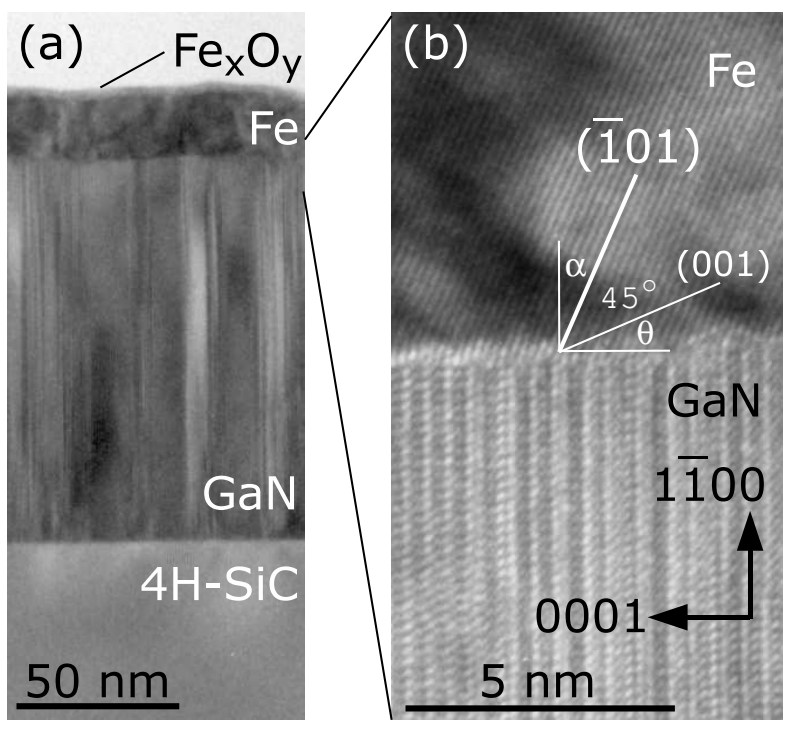

FIG. 1: (a) Cross-sectional transmission electron micrograph taken along the $[\overline{1120}]$ zone axis of the $\mathrm{Fe} / \mathrm{GaN} / 4 \mathrm{H}-\mathrm{SiC}$ structure under investigation. The top $2 \mathrm{~nm}$ of the Fe film are oxidized. (b) Cross-sectional high-resolution transmission electron micrograph of the $\mathrm{Fe} / \mathrm{GaN}(1 \overline{1} 00)$ interface along the [1120] zone axis. The $\mathrm{Fe}(\overline{101})$ and $\mathrm{GaN}(0001)$ lattice planes form an angle, $\alpha$, of approximately $23^{\circ}$. Stacking faults in the GaN, visible in both panels, do not affect the orientation of the Fe film.

tering diffraction (EBSD) was carried out in a Zeiss Ultra55 scanning electron microscope equipped with an EDAXTSL EBSD system.

Figure 1 a) shows a cross-sectional transmission electron micrograph of the Fe/GaN/4H-SiC structure. Despite the structural and chemical differences of the constituent materials, the respective layers are well defined and exhibit abrupt interfaces. The GaN layer is seen to contain stacking faults due to the stacking mismatch between $2 \mathrm{H}$ $\mathrm{GaN}$ and $4 \mathrm{H}-\mathrm{SiC}$. Nevertheless, the high-resolution detail 


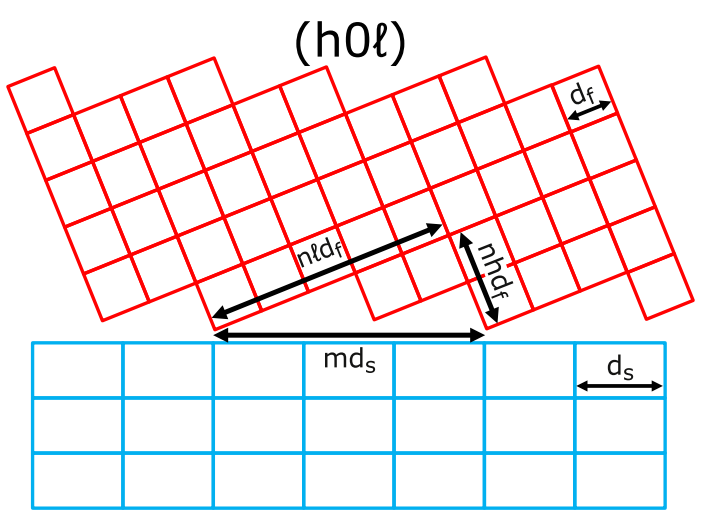

FIG. 2: Schematic view of a commensurate interface between a film with lattice constant $d_{f}$ and a substrate with lattice constant $d_{s}$. The interface plane is $(h 0 \ell)$ with respect to the film, corresponding to a tilting from (001) toward (101) by the angle $\theta=\tan ^{-1}(h / \ell)$. The condition for commensurability is that a coincidence-site lattice, defined by a pair of integers $(m, n)$, exists such that $m d_{s}=n\left(h^{2}+\ell^{2}\right)^{1 / 2} d_{f}$. The example depicted here is (205) with $\theta=21.8^{\circ}$ (equivalent to the angle $\alpha=23.2^{\circ}$ as defined in Fig. 1) and $(m, n)=(3,1)$.

of the $\mathrm{Fe} / \mathrm{GaN}$ interfacial region shown in Fig.1 b) reveals an epitaxial relationship between the Fe film and the underlying $\mathrm{GaN}(1 \overline{1} 00)$ layer. The (101) lattice planes of the $\mathrm{Fe}$ film are clearly resolved and are found to be well ordered, unaffected by the stacking disorder in the GaN layer. Of special interest is the angle, approximately $23^{\circ}$, formed by the Fe (101) planes and the vertical interface normal. This angle indicates that the Fe interface plane has a high Miller index - an unexpected finding in light of the comparatively high surface energies of high-index metal surfaces. We show now that precisely this orientation is predicted by a simple, physically transparent model (complemented with first-principles total-energy calculations) of epitaxial interfaces between dissimilar materials.

Consider the formation of an interface between a film $(f)$ and a substrate $(s)$ having different lattice constants $d_{f}$ and $d_{s}$. If the lattice mismatch is sufficiently small then the strained film may grow coherently until it reaches its critical thickness [3]. For much larger mismatch this scenario becomes unlikely. Epitaxial growth is nevertheless possible by tilting the orientation of the film, as the TEM image in Fig. 1 b) makes clear. Figure 2 illustrates how an arbitrary lattice mismatch can be accommodated by allowing the film to have an orientation between (001) and (101) given by the Miller indices $(h 0 \ell)$. Our goal below is to predict the most stable film orientation given the lattice constants $d_{f}$ and $d_{s}$.

The unit cell of a film with orientation $(h 0 \ell)$ has length $L_{f}=\left(h^{2}+\ell^{2}\right)^{1 / 2} d_{f}$. In order for the film and substrate to be commensurate there must exist a coincidence-site

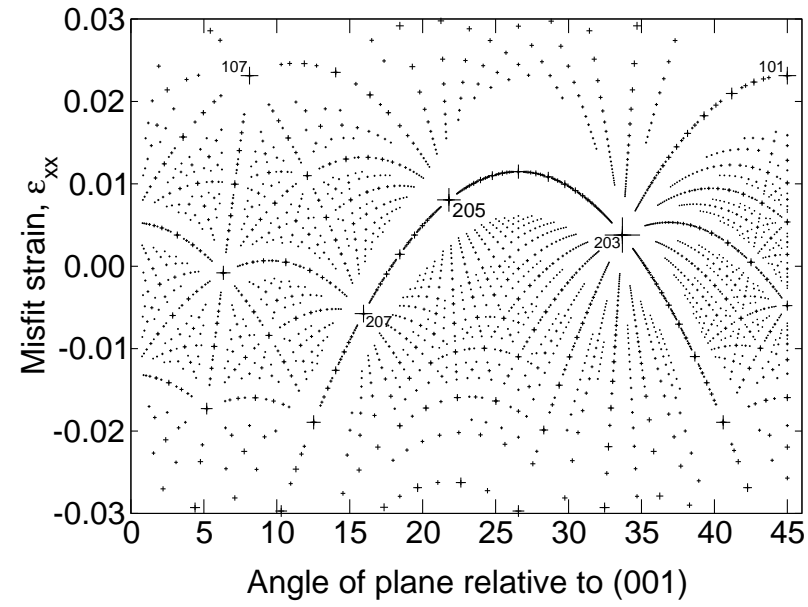

FIG. 3: Misfit strain in commensurate interfaces between bcc Fe and $\mathrm{GaN}(1 \overline{1} 00)$. The points represent all possible $\mathrm{Fe}(h 0 \ell)$ planes having $h \leq \ell \leq 75$. The misfit $\varepsilon_{x x}$ is the strain component along the [0001] direction of the GaN. The size of each plot symbol is inversely proportional to the period $m$ of the coincidence-site lattice (CSL) that minimizes the misfit. $\mathrm{Fe}(h 0 \ell)$ planes having both small misfit strain and small CSL period are labeled.

lattice (CSL), defined by a pair of integers $(m, n)$, such that $m d_{s}=n L_{f}$. This condition is unrealistically restrictive, however. In real systems the film will tolerate a small compressive or tensile strain $\varepsilon_{x x}$ which relaxes the CSL condition to $m d_{s}=n L_{f}\left(1+\varepsilon_{x x}\right)$.

We propose two geometrical criteria for identifying candidate orientations for interfaces with low energy. (1) The misfit strain $\varepsilon_{x x}$ should be as small as possible, and (2) the period $m$ of the CSL should be as small as possible. The latter criterion is motivated by analogy to low-energy grain boundaries between two identical materials, which often have a CSL with small unit cell volume $\Sigma$ [4]. For interfaces between different materials it is not generally possible to minimize the strain and CSL period simultaneously. Nor is it obvious how to construct a single objective function of both which could then be optimized. Instead, we apply both criteria with the aim of selecting a small subset of candidate orientations for subsequent study with a more quantitative method such as density-functional theory (DFT).

To apply these criteria to the growth of Fe on the $M$ plane of GaN we equate $d_{f}$ with the bcc Fe lattice constant, $2.866 \AA$ [7], and $d_{s}$ with the GaN $c$ lattice parameter, 5.186 $\AA$ [8]. Figure 3 shows the resulting Fe misfit strain needed to satisfy the CSL condition for a large number of hypothetical orientations of the $\mathrm{Fe}$ film. In this plot each orientation $(h 0 \ell)$ is represented by its angle $\theta=\tan ^{-1}(h / \ell)$ relative to the (001) plane. The period of each CSL is encoded by the size of the plot symbol, which is inversely 


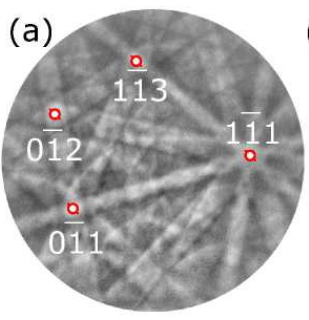

(b)
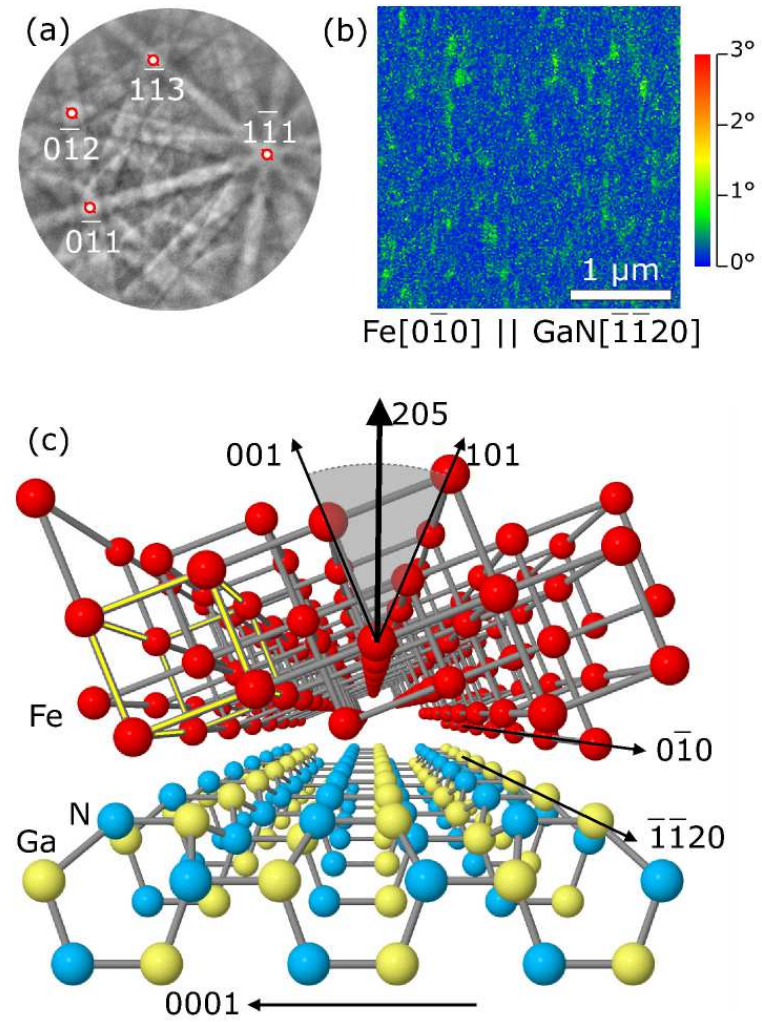

FIG. 4: (a) Pseudo-Kikuchi pattern of the Fe film on GaN(11 00 ) depicted in Fig. 11 The major low-index zone axes are indicated. (b) In-plane orientation map of the Fe film. Variations in color encode local deviations from the nominal orientation. The film has the same orientation over the entire area within a tolerance of $1^{\circ}$. (c) Model of the $\mathrm{Fe}(205) / \mathrm{GaN}(1 \overline{1} 00)$ epitaxial interface. For clarity an ideal geometry with arbitrary registry is shown here. Slightly less than one unit cell along [0001] is depicted. A conventional unit cell of $\mathrm{Fe}$ is highlighted in yellow.

proportional to $m$. Only points with small strains, less than $3 \%$, are displayed here.

The rich structure visible in Fig. 3 makes it clear that the misfit strain can be made arbitrarily small for many different film orientations. Hence the strain alone cannot provide a definitive criterion favoring a particular growth plane. Moreover, the vast majority of these low-strain orientations require a very large CSL period and hence do not constitute physically meaningful commensurability. Only a very few orientations offer both a small strain and small CSL period, namely (207), (205), and (203). The (205) orientation corresponds to the angle $\theta=21.8^{\circ}$. This is equivalent to $\alpha=45-\theta=23.2^{\circ}$ as defined in Fig. 1(b) and thus is in excellent agreement with the measured angle, $23^{\circ}$, obtained from TEM.

It is important to realize that our purely geometrical criteria do not distinguish between the orientation (205) de- picted in Fig. 2 and its symmetry-equivalent counterpart, $(20 \overline{5})\left(\theta=-21.8^{\circ}\right)$, created by rotating the film by $180^{\circ}$ about the substrate normal. Indeed, the pairs $[(h 0 \ell),(h 0 \bar{\ell})]$ have the same strain and CSL and hence are equivalent within this model. In the $\mathrm{Fe} / \mathrm{GaN}$ system, however, the polarity of the GaN wurtzite structure breaks this equivalence. The question that then arises is whether the influence of the polarity is sufficiently strong to select a single orientation, and if so, which one? The TEM image in Fig. 1 shows a single orientation but is limited to a nanometer-scale region of the film. To characterize a much larger area we used EBSD [9]. Figure 4(a) shows the resulting pseudo-Kikuchi pattern of the Fe film in Fig.11 The pattern exhibits sharp and well defined Kikuchi bands, reflecting high crystal quality of the Fe film and allowing for a fast and reliable indexing of the patterns recorded while scanning the electron beam over a large area $\left(9 \mu \mathrm{m}^{2}\right)$. The resulting EBSD map shown in Fig. 4(b) visualizes the inplane orientation of the Fe film with a spatial and angular resolution of $20 \mathrm{~nm}$ and $1^{\circ}$, respectively. The map reveals the complete absence of any domain structure. Indeed, the film is single crystalline, and has the same orientation as found by TEM throughout the mapped area.

To understand why a single orientational domain is found requires going beyond a model based solely on interface geometry. Now the interface structure-its precise atomic arrangement and chemical bonding-must be addressed. To do this we used DFT to calculate the relative formation energies of finite $\mathrm{Fe}$ films grown on the $M$-plane of $\mathrm{GaN}$. We considered the six different orientations predicted by the geometrical model to be favorable: (207),

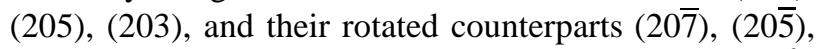
$(20 \overline{3})$. The different films were the same thickness, $6 \AA$, equivalent to about four monolayers. Figure 4(c) depicts the $\mathrm{Fe}(205) / \mathrm{GaN}(1 \overline{1} 00)$ interface as an example.

We have previously shown that at 3-4 monolayers the contribution of the $\mathrm{Fe} / \mathrm{GaN}$ interface formation energy to the full formation energy of the film is already converged [10]. We also find that the $\mathrm{Fe}$ free surface energy varies by less than $1 \mathrm{meV} / \AA^{2}$ among the three orientations we consider here [11]. Therefore the relative formation energy of the finite film closely mirrors, with good accuracy, the formation energy of the isolated interface.

For each orientation the Fe film was slightly strained along the $x[\mathrm{GaN}(0001)]$ direction according to its CSL as discussed above. There is also a lattice mismatch in the $y[\mathrm{GaN}(\overline{11} 20))]$ direction because the Fe lattice constant and GaN $a$ lattice parameter differ by nearly $12 \%$. This mismatch was accommodated by a single CSL, common to all orientations, containing eight unit cells of $\mathrm{Fe}$ and seven of GaN. The GaN substrate was represented by a slab of four atomic layers with fixed in-plane equilibrium lattice parameters and a passivating bottom layer. 
TABLE I: Formation energies calculated within densityfunctional theory of $\mathrm{Fe} / \mathrm{GaN}(1 \overline{1} 00)$ films for the $\mathrm{Fe}$ planes in Fig. 3 that have both a small misfit strain and a coincidence-site lattice (CSL) with small period $m$. The Fe film thickness is the same for all cases, approximately $6 \AA$. Formation energies are relative to the most favorable plane, $\mathrm{Fe}(205)$, in units of $\mathrm{meV} / \AA^{2}$.

\begin{tabular}{cccc}
\hline \hline Plane & Misfit strain & CSL period & Formation energy \\
\hline $\mathrm{Fe}(205)$ & +0.008 & 3 & 0 \\
$\mathrm{Fe}(20 \overline{5})$ & +0.008 & 3 & 4 \\
& & & 9 \\
$\mathrm{Fe}(203)$ & +0.004 & 2 & 16 \\
$\mathrm{Fe}(20 \overline{3})$ & +0.004 & 2 & \\
$\mathrm{Fe}(207)$ & -0.006 & 4 & 12 \\
$\mathrm{Fe}(20 \overline{7})$ & -0.006 & 4 & 13 \\
\hline \hline
\end{tabular}

Total energies and forces were calculated within the PBE generalized-gradient approximation [12] to DFT using projector-augmented-wave potentials as implemented in VASP [13, 14]. All Fe and $\mathrm{GaN}$ atomic positions were fully relaxed except the bottom $\mathrm{GaN}$ layer. For each $\mathrm{Fe}$ film orientation the formation energy depends strongly on the choice of $\mathrm{Fe}-\mathrm{GaN}$ interface registry. We systematically varied the registry over a grid in both $x$ and $y$ to locate the global energy minimum for each orientation. The planewave cutoff for all calculations was $400 \mathrm{eV}$.

The resulting formation energies are listed in Table [) The most favorable orientation is $\mathrm{Fe}(205)$. Of the candidates tested, this orientation has neither the smallest possible strain nor the shortest possible CSL period, indicating that the optimal interface structure is an important third criterion that must supplement the two geometrical criteria. Note also that the small variation in the formation energy of the Fe surface, which is included in the formation energy of the film, is too small to affect the overall energy ordering of the orientations.

To distinguish $\mathrm{Fe}(205)$ from $\mathrm{Fe}(20 \overline{5})$ experimentally requires determining the absolute polarity of the GaN substrate. We did this by recording CBED patterns with a beam spot size of approximately $15 \mathrm{~nm}$ under two-beam conditions. Simulations of CBED patterns were performed using JEMS [15] to index the crystallographic directions observed in the experimental patterns and thus to determine the polarity. The resulting absolute orientation relationship is $\mathrm{Fe}(205)[0 \overline{1} 0]|| \mathrm{GaN}(1 \overline{1} 00)[\overline{11} 20]$, in agreement with the prediction of our theoretical model complemented with the results of DFT calculations.

Our model suggests a new strategy for growing non- polar GaN films. The basic idea is to turn Fig. 2 upsidedown and consider the growth of $M$-plane GaN on a suitable high-index substrate. For a given material this requires identifying candidate orientations corresponding to small strain and small CSL period. One very promising material is $\mathrm{Si}$, which is already in widespread use as a flat substrate for GaN/Si epitaxy despite the resulting high dislocation densities [16]. Many high-index Si substrates are readily available, and some have already been used for $\mathrm{GaN}$ growths [17, 18]. Calculations are in progress to identify promising high-index $\mathrm{Si}$ orientations for growing nonpolar $\mathrm{GaN}$ with low strain [19].

[1] E. G. Bauer, B. W. Dodson, D. J. Ehrlich, L. C. Feldman, C. P. Flynn, M. W. Geis, J. P. Harbison, R. J. Matyi, P. S. Peercy, P. M. Petroff, et al., J. Mater. Res. 5, 852 (1990).

[2] C. J. Palmstrøm, Ann. Rev. Mat. Sci. 25, 389 (1995).

[3] J. W. Matthews and A. E. Blakeslee, J. Crystal Growth 27, 118 (1974).

[4] A. P. Sutton and R. W. Balluffi, Acta Metall. 35, 2177 (1987).

[5] A. Trampert, Physica E 13, 1119 (2002).

[6] O. Brandt, Y. J. Sun, L. Däweritz, and K. H. Ploog, Phys. Rev. B 69, 165326 (2004).

[7] H. K. Mao, W. A. Bassett, and T. Takahashi, J. Appl. Phys. 38, 272 (1967).

[8] M. Leszczynski, H. Teisseyre, T. Suski, I. Grzegory, M. Bockowski, J. Jun, S. Porowski, K. Pakula, J. M. Baranowski, C. T. Foxon, et al., Appl. Phys. Lett. 69, 73 (1996).

[9] G. Nolze, V. Geist, R. S. Neumann, and M. Buchheim, Cryst. Res. Technol. 40, 791 (2005).

[10] C. X. Gao, O. Brandt, S. C. Erwin, J. Lähnemann, U. Jahn, B. Jenichen, and H. P. Schönherr, Phys. Rev. B 82, 125415 (2010).

[11] S. C. Erwin (unpublished).

[12] J. P. Perdew, K. Burke, and M. Ernzerhof, Phys. Rev. Lett. 77, 3865 (1996).

[13] G. Kresse and J. Hafner, Phys. Rev. B 47, 558 (1993).

[14] G. Kresse and J. Furthmüller, Phys. Rev. B 54, 11169 (1996).

[15] P. A. Stadelmann, JEMS electron microscopy software (Java version 3.5110U2010), CIME-EPFL, Switzerland, 1999-2010.

[16] S. Joblot, Y. Cordier, F. Semond, S. Chenot, P. Vénnègues, O. Tottereau, P. Lorenzini, and J. Massies, Superlattices and Microstructures 40, 295 (2006).

[17] X. Ni, M. Wu, J. Lee, X. Li, A. A. Baski, Ü. Özgür, and H. Morkoç, Appl. Phys. Lett. 95, 111102 (2009).

[18] R. Ravash, J. Blaesing, A. Dadgar, and A. Krost, Appl. Phys. Lett. 97, 142102 (2010).

[19] A. Kutana and S. C. Erwin (unpublished). 